\title{
Development and validation of algorithms for heart failure patient care: a Delphi study
}

\author{
Cynthia Priyadarshini Gopal ${ }^{1}$, BPharm, MPharm, Asri Ranga² ${ }^{2}$ MD, MMed, Kevin Louis Joseph ${ }^{3}$, MBBS, MRCP,
} Balamurugan Tangiisuran ${ }^{1}$, MPharm, PhD

INTRODUCTION Although heart failure (HF) management is available at primary and secondary care facilities in Malaysia, the optimisation of drug therapy is still suboptimal. Although pharmacists can help bridge the gap in optimising HF therapy, pharmacists in Malaysia currently do not manage and titrate HF pharmacotherapy. The aim of this study was to develop treatment algorithms and monitoring protocols for angiotensin-converting enzyme inhibitors, angiotensin II receptor blockers, beta-blockers and spironolactone based on extensive literature review for validation and utilisation by pharmacists involved in HF management.

METHODS A Delphi survey involving 32 panellists from private and government hospitals that provide cardiac services in Malaysia was conducted to obtain a consensus of opinion on the treatment protocols. The panellists completed two rounds of self-administered questionnaires to determine their level of agreement with all the components in the protocols. RESULTS Consensus was achieved for most of the sections of the protocols for the four classes of drugs. The panellists' opinions were taken into consideration when amending the components of the protocols that did not achieve consensus of opinion. Full consensus was achieved with the second survey conducted, enabling the finalisation of the drug titration protocols. CONCLUSION The resulting validated HF titration protocols can be used as a guide for pharmacists when recommending the initiation and titration of HF drug therapy in daily clinical practice. Recommendations should be made in collaboration with the patient's treating physician, with concomitant monitoring of the patient's response to the drugs.

Keywords: Delphi study, heart failure, monitoring protocols, pharmacist, treatment algorithms

\section{INTRODUCTION}

Heart failure (HF) is the terminal consequence of many cardiac disorders, and the incidence of $\mathrm{HF}$ is expected to increase exponentially in the future. ${ }^{(1)}$ Although there are available pharmacotherapies that improve the mortality and morbidity of $\mathrm{HF}^{(2-5)}$ these pharmacotherapies are often underutilised or prescribed at suboptimal doses. ${ }^{(6,7)}$ To address this shortcoming, many developed countries have initiated and established specialised HF clinics that are managed by a multidisciplinary team comprising physicians, pharmacists and various paramedical staff. Pharmacist-recommended optimisation of angiotensin-converting enzyme (ACE) inhibitor therapy has been shown to result in fewer hospitalisations and lower treatment costs. ${ }^{(8-10)}$ When pharmacists initiated and up-titrated anti-failure medications under the supervision of a cardiologist, the prescription rates of ACE inhibitors, beta-blockers and spironolactone increased, with improvements in the symptoms and functional class of patients (i.e. reductions in heart rate and systolic blood pressure without adverse renal effects). ${ }^{(11)}$

In Malaysia, specialised HF clinics are not available in all government and private hospitals. Thus, many patients with HF are managed at a primary and secondary care level, often by junior medical staff. In such cases, pharmacist involvement in HF patient care can be enhanced through the use of standardised evidencebased treatment protocols. Hence, the primary objective of this study was to develop HF treatment algorithms and monitoring protocols and to validate them using an adapted Delphi method.

\section{METHODS}

Randomised controlled trials, meta-analyses and review articles on HF management and guidelines, which were published between January 1998 and December 2012, were identified using PubMed, OvidSP, SpringerLink, ScienceDirect and EBSCOhost. Using predefined search criteria, a total of 3,142 articles were identified. Of the 320 articles subsequently selected for full-text reading, 212 articles were deemed beneficial to this study. The evidence-based data obtained from this literature review was used to develop detailed guidance on initiating, titrating and monitoring ACE inhibitors, angiotensin II receptor blockers (ARBs), beta-blockers and spironolactone.

The treatment protocols were then validated using an adapted Delphi survey. The Delphi survey technique was used to generate discussion among a panel of experts and to eventually obtain a consensus of opinion regarding HF management. This technique enables information and opinions to be collected in a neutral environment. ${ }^{(12)}$ Since information and opinions were gathered via several rounds of structured questionnaires, face-to-face meeting of the panellists was avoided. This maintains the anonymity of the responses and prevents monopolisation of any particular opinion. In this study, two rounds of surveys were conducted on the panel of experts. The responses of the panellists were tallied and amendments were made before the protocols were ready to be used.

To recruit the panel of experts for the present study, we invited 40 doctors and pharmacists from local private, institutional

${ }^{1}$ School of Pharmaceutical Sciences, Universiti Sains Malaysia, Pulau Pinang, ${ }^{2}$ Hospital Raja Permaisuri Bainun, ${ }^{3}$ Hospital Pantai, Ipoh, Perak, Malaysia Correspondence: Dr Balamurugan Tangiisuran, Senior Lecturer, School of Pharmaceutical Sciences, Universiti Sains Malaysia, 11800 Minden, Pulau Pinang, Malaysia. bala@usm.my 
and government hospitals via email and telephone. Of these 40 doctors and pharmacists, 32 agreed to participate in the study; the remaining 8 declined due to work commitments or without reason. The final panel consisted of 12 cardiologists, 10 physicians and 10 pharmacists. Among the 32 panellists, half were women. On average, the pharmacists were about ten years younger than the cardiologists and physicians. Most of the panellists $(71.9 \%$ ) had over ten years of clinical experience, and at least half of the patients they managed monthly were patients with HF.

The panellists were required to complete a self-administered questionnaire, which aimed to determine their level of agreement with the ACE inhibitor, ARB, beta-blocker and spironolactone treatment protocols in relation to the following areas: (a) contraindications and cautions to initiating drug therapy; (b) individual and overall components of initiation and titration algorithms; (c) monitoring parameters and frequency of monitoring; (d) recommended approaches to dealing with commonly encountered treatment-related problems; (e) information to be provided to patients; and ( $f$ ) clinically significant interactions between the four agents and commonly co-administered drugs. The level of agreement was measured using a Likert scale (1-9). A score of 1, 2, 3 or 4 indicated varying degrees of disagreement with the statement/question - 1 indicated the highest level of disagreement and 4 , the lowest level of disagreement. A score of $6,7,8$ or 9 indicated varying degrees of agreement -6 indicated the lowest level of agreement and 9, the highest level of agreement. A score of 5 represented a neutral opinion. The panellists were also encouraged to provide constructive comments.

The survey questionnaires were emailed $(n=4)$, posted ( $n=3$ ) or hand-delivered $(n=25)$. In the first round, the panel of experts received a formal letter of invitation, a study information sheet, the survey questionnaire and a self-addressed return envelope (if applicable). Confirmation of receipt of the questionnaires was done three days after mailing via telephone and email. A telephone reminder and a one-week extension were given for panellists who did not return the questionnaires on the due date, in view of their busy work schedules. Once all completed questionnaires were received, the responses were collated and tallied. Questions that achieved a group consensus were not presented for discussion in the second round, while questions that did not achieve a group consensus in the first round were re-presented in the second round, but only to the panellists whose responses were outside the majority opinion. Amendments to treatment protocols were made according to the suggestions of the panellists, prior to the second survey. The entire process of the Delphi method used in the present study is illustrated in Fig. 1.

Data collected were entered and tabulated using Microsoft Excel (Microsoft Corp, Redmond, WA, USA). Descriptive statistics were generated for each designated Delphi question to determine whether a consensus had been achieved. A 'consensus of opinion' was defined as $\geq 70 \%$ agreement among the panellists; a 'majority opinion' was defined as $51 \%-69 \%$ agreement among the panellists, while a 'minority opinion' was defined as $\leq 50 \%$ agreement among the panellists. ${ }^{(13,14)}$

\section{RESULTS}

The panellists reached a consensus for all questions relating to: (a) contraindications to the commencement of the four agents (i.e. ACE inhibitors, ARBs, beta-blockers and spironolactone); and (b) cautions for initiating three of the four drugs in patients with HF (only a majority opinion was achieved for two of the five cautions for initiating beta-blocker therapy). With regard to the cautions for initiating beta-blocker therapy, 10 (31.3\%) of the panellists disagreed on commencing beta-blockers among patients with hepatic and renal diseases. One panellist reasoned that this was because the statement was vague. Upon further explanation, seven of the ten panellists who initially disagreed joined the majority opinion, allowing a consensus to be achieved. There were also differing opinions (11 disagreed, 1 neutral) about the initiation of beta-blockers in patients with diabetes mellitus, as there was concern that such treatment would mask hypoglycaemic symptoms. After this statement was reworded, without compromising its integrity, a consensus was achieved in the second survey.

With regard to the individual drug algorithms, two sections of the ACE inhibitor and ARB treatment algorithms achieved a majority opinion, while the other sections achieved consensus. All the physicians $(n=10)$ surveyed indicated that they were not satisfied with the titration algorithms for ACE inhibitors (Fig. 2) and ARBs (Fig. 3), in cases where serum creatinine levels increased by $30 \%$ to $<50 \%$ and $50 \%$ to $<100 \%$. This portion was deemed too complicated for pharmacists to use, and drug cessation was considered necessary and warranted once serum creatinine level increased by more than $30 \%$ from the baseline. As all the physicians $(n=10)$ were not agreeable to this portion of the algorithm, this component of the titration protocol was amended to achieve consensus in the second survey. First-round consensus was achieved for the individual titration algorithms for beta-blockers and spironolactone (Figs. 4 \& 5).

In the third section of the questionnaire, which covered monitoring parameters and frequency, only $62.5 \%$ agreed (i.e. a majority opinion) with monitoring of electrolytes and renal function when using beta-blockers. A similar result was found for all but one of the parameters relating to information to be provided to the patient and clinically significant drug interactions with commonly used HF medications. When assessing the 'troubleshooting' section of the protocols, consensus was achieved among the panellists with regard to the suggested methods to deal with problems related to treatment with ACE inhibitors, ARBs, beta-blockers and spironolactone.

\section{DISCUSSION}

The present study showed that there were some concerns regarding the definitions of renal and hepatic failure when beta-blocker therapy is being considered for patients with HF. Post hoc analyses revealed that the addition of beta-blockers to standard HF therapy for patients with moderate to severe 


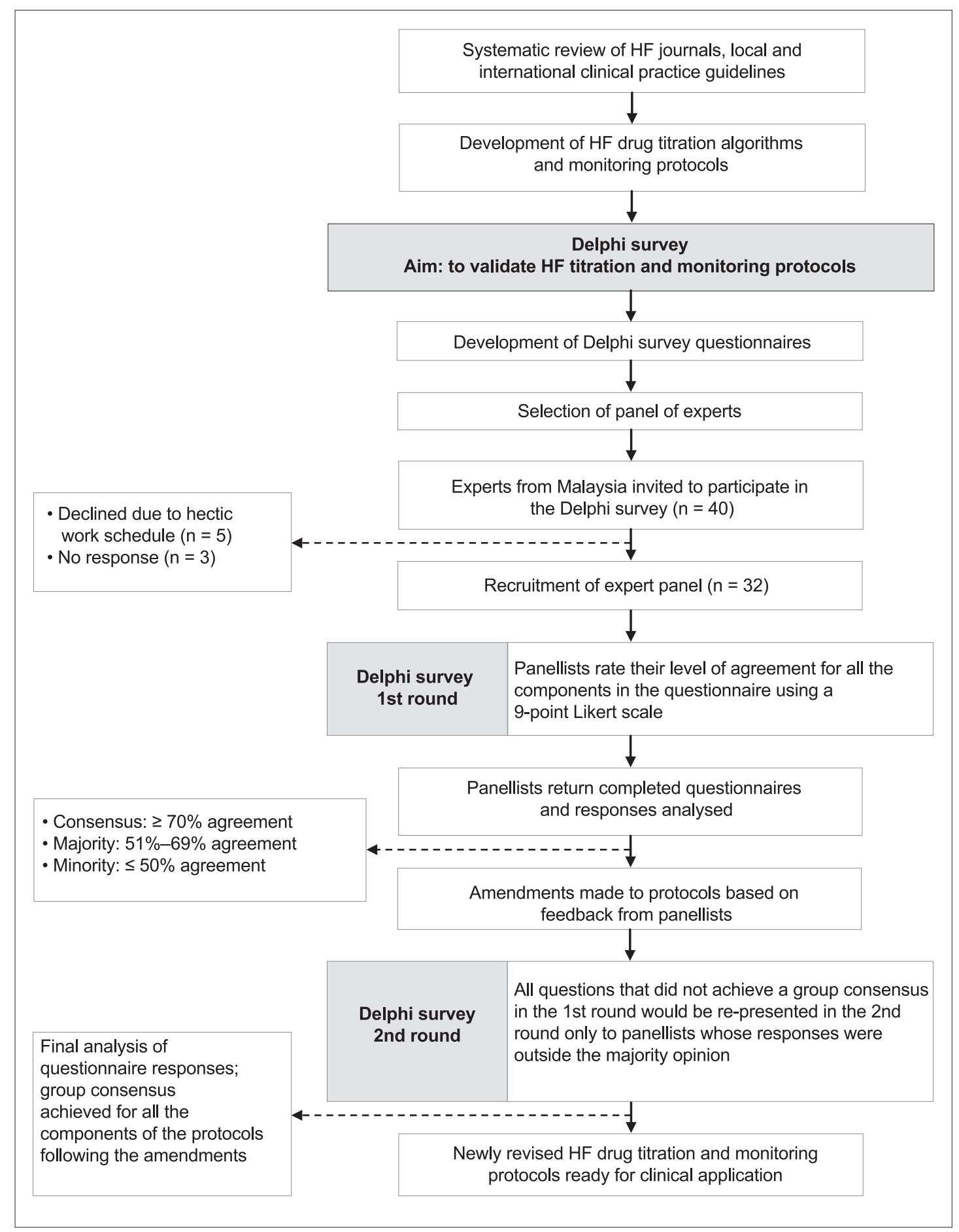

Fig. 1 Flowchart shows the process of the Delphi method used in the present study. HF: heart failure

renal impairment (i.e. serum creatinine $>300 \mu \mathrm{mol} / \mathrm{L}$ ) was found to reduce all-cause mortality in the Cardiac Insufficiency Bisoprolol Study II, ${ }^{(15)}$ while for patients with worse renal function (estimated glomerular filtration rate $<45 \mathrm{~mL} / \mathrm{min} / \mathrm{m}^{2}$ using the Modification of Diet in Renal Disease formula), greater mortality benefits (hazard ratio 0.41 ) were noted in the Metoprolol CR/XL Randomized Intervention Trial in Chronic HF. ${ }^{(16)}$ Mortality and HF admissions were also found to be significantly reduced in a small study that used carvedilol in patients with dilated cardiomyopathy on regular haemodialysis. ${ }^{(17)}$ As renal impairment is an independent predictor of negative outcomes in HF and beta-blocker therapy is beneficial in HF, it was concluded that patients at all levels of renal impairment would benefit from beta-blocker therapy, although clinicians should exercise caution. ${ }^{(15-17)}$ With respect to liver impairment, metoprolol and carvedilol undergo extensive first-pass metabolism, whereas bisoprolol is excreted equally via the kidneys and liver. ${ }^{(18)}$ Carvedilol elimination is significantly prolonged in patients with moderate to severe liver cirrhosis, warranting a two- to threefold reduction of the drug dose. ${ }^{(19)}$ In view of this, beta-blockers can be utilised in patients with liver and renal impairment, albeit cautiously. 


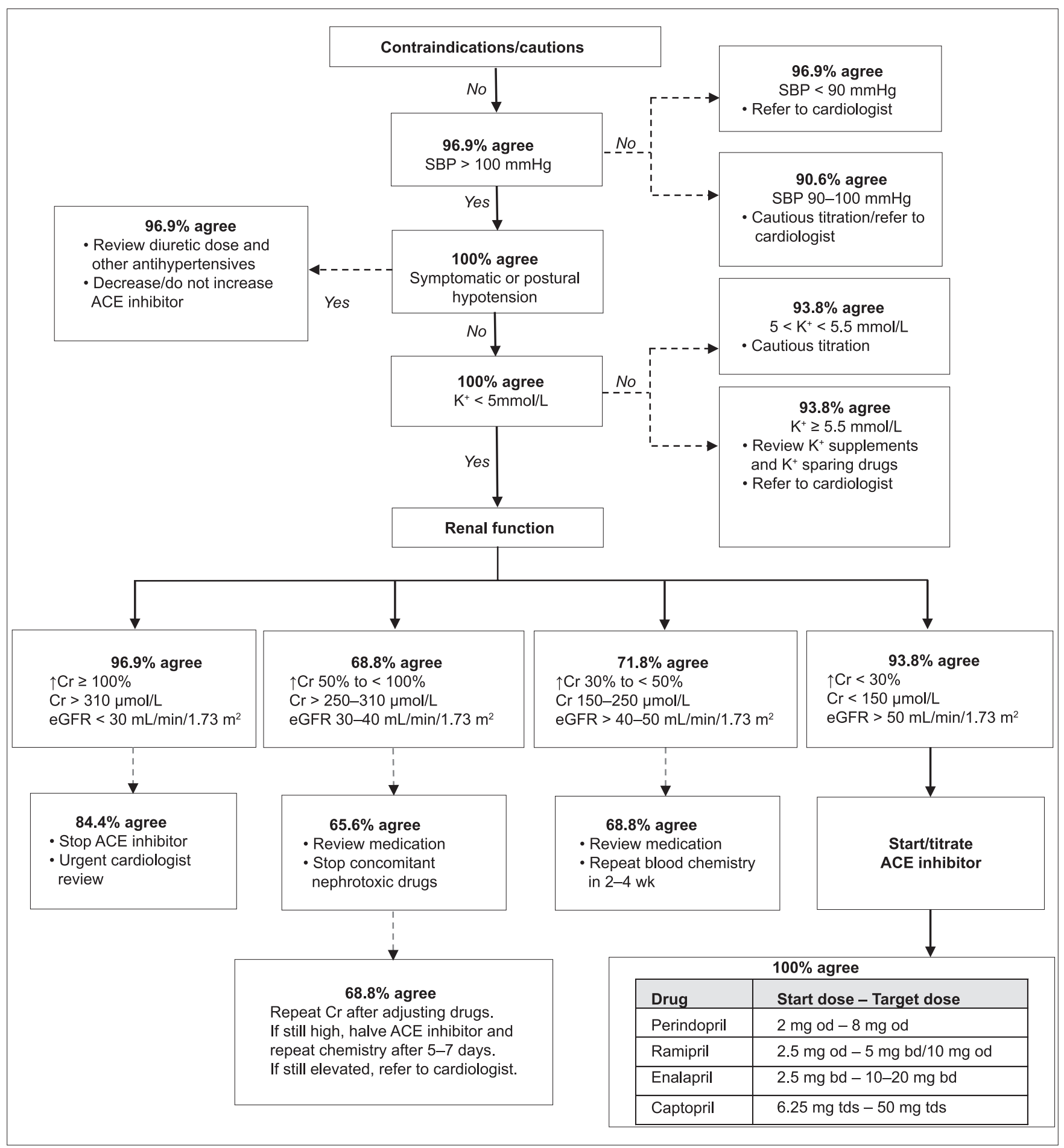

Fig. 2 Flowchart shows the percentage of agreement among the panellists for the angiotensin-converting enzyme (ACE) inhibitor initiation/titration algorithm, following the first round of the Delphi survey. bd: twice daily; Cr: serum creatinine; eGFR: estimated glomerular filtration rate using the Modification of Diet in Renal Disease formula; $\mathrm{K}^{+}$: serum potassium; od: once daily; SBP: systolic blood pressure; tds: three times daily

A point of contention for all the physicians in the present study was the renal parameter cut-off levels for referral and uptitration of ACE inhibitors and ARBs. The cardiologists in the present study were comfortable with close monitoring instead of drug cessation in patients whose serum creatinine level rose more than $30 \%$ from baseline. In a study conducted by Bakris et al, the commencement of ACE inhibitors and ARBs in patients with pre-existing renal impairment (serum creatinine $\geq 124$ $\mu \mathrm{mol} / \mathrm{L}$ ) reduced the progression of renal disease by $55 \%-75 \%$. ${ }^{(20)}$
Although the serum creatinine levels of the patients in that study were up to $30 \%$ higher than the baseline, the levels then stabilised within two months of drug initiation. ${ }^{(20)}$ On the other hand, physicians were been found to be generally more conservative, and thus, 1.1-2.5 times less likely to prescribe ACE inhibitors than cardiologists. ${ }^{(21)}$ Cardiologists were found to be more likely to prescribe higher doses of ACE inhibitors and less likely to preclude its use in patients with renal impairment (i.e. serum creatinine $>2.5 \mathrm{mg} / \mathrm{dL}$ or $221 \mu \mathrm{mol} / \mathrm{L}) .{ }^{(22)}$ Physicians with more recent training 


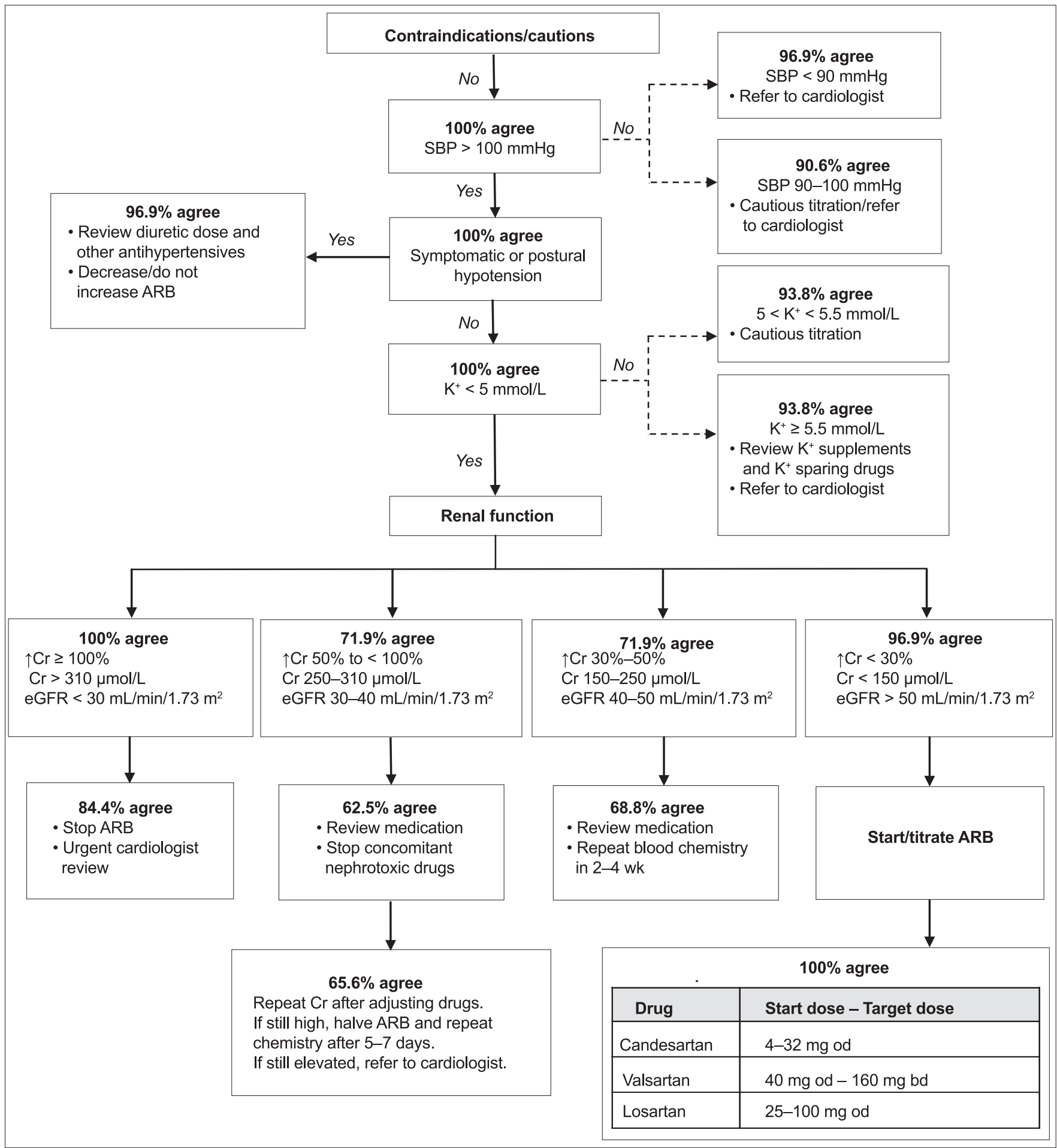

Fig. 3 Flowchart shows the percentage of agreement among the panellists for the angiotensin II receptor blocker (ARB) initiation/titration algorithm, following the first round of the Delphi survey. bd: twice daily; Cr: serum creatinine; eGFR: estimated glomerular filtration rate using the Modification of Diet in Renal Disease formula; $\mathrm{K}^{+}$: serum potassium; od: once daily; SBP: systolic blood pressure

were shown to have practice patterns that were more similar to those of cardiologists, as compared to physicians with more distant training. ${ }^{(22)}$ The comments made by the physicians in the present study may be reflective of the aforementioned finding, as the majority of them (> 80\%) had worked for more than ten years.

As the protocols developed in our study are intended for the use of pharmacists, who possibly have less clinical experience than our panellists and who are situated in centres with few or no physicians/Family Medicine Specialist (FMS), minor changes were made to the ACE inhibitor and ARB titration protocols, such that early referral to a cardiologist/physician/FMS is encouraged when serum creatinine level rises above $30 \%$ from the baseline. Review of concomitant nephrotoxic drugs and close biochemical monitoring, with or without cessation of ACE inhibitors and ARBs, are advised when creatinine level rises by $50 \%$ to $<100 \%$ and when delayed specialist review was anticipated.

In the present study, all the physicians $(\mathrm{n}=10)$ and two of the cardiologists felt that monitoring renal function was not necessary 


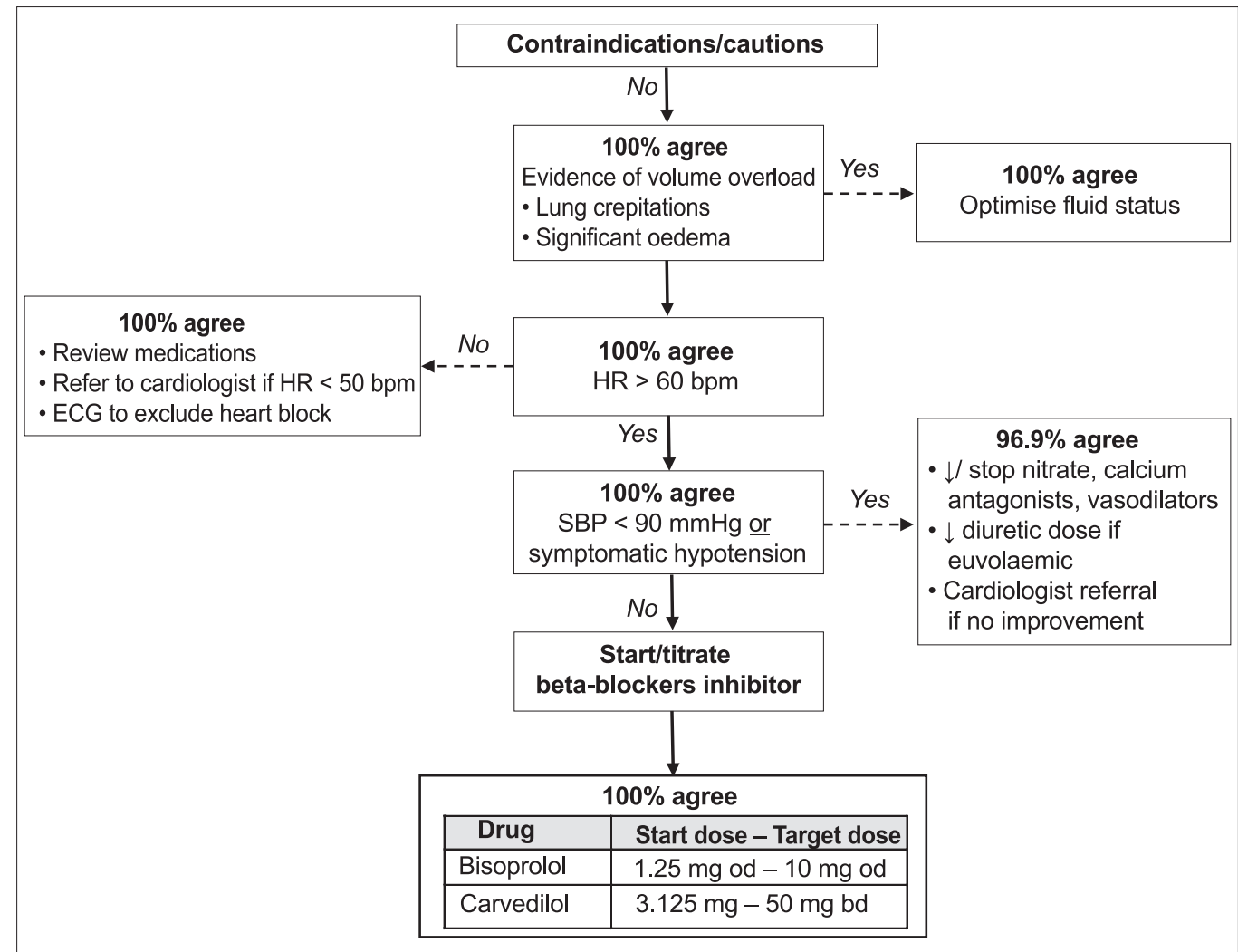

Fig. 4 Flowchart shows the percentage of agreement among the panellists for the beta-blocker initiation/titration algorithm, following the first round of the Delphi survey. bd: twice daily; ECG: electrocardiogram; HR: heart rate; od: daily; SBP: systolic blood pressure

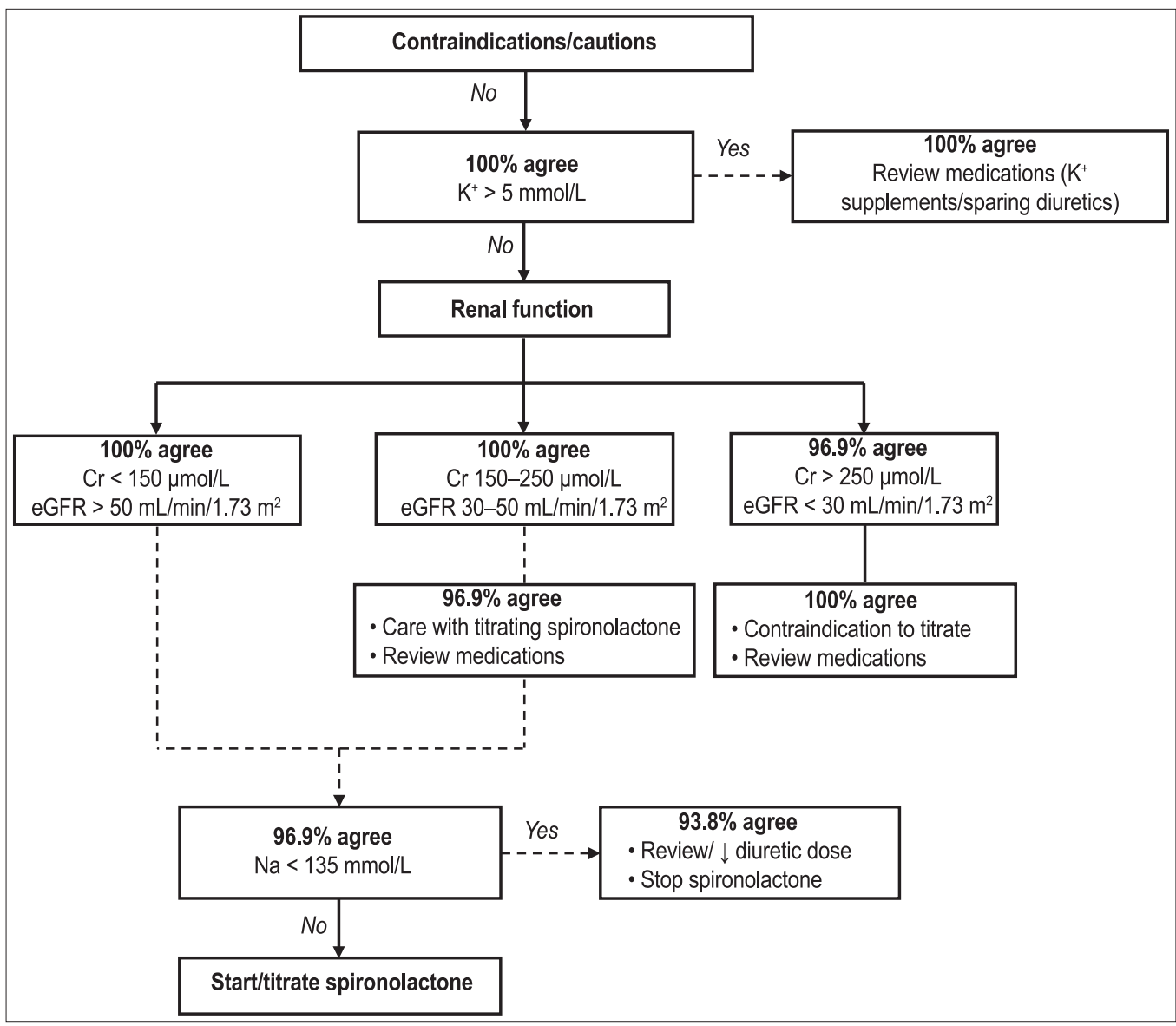

Fig. 5 Flowchart shows the percentage of agreement among the panellists for the spironolactone initiation/titration algorithm, following the first round of the Delphi survey. Cr: serum creatinine; eGFR: estimated glomerular filtration rate using the Modification of Diet in Renal Disease formula; ${ }^{+}$: serum potassium; Na: serum sodium 
when titrating beta-blockers. This parameter was included, as it was one of the expert recommendations listed in the National Institute for Health and Clinical Excellence guidelines on chronic HF management. ${ }^{(23)}$ Beta-blockers may transiently worsen HF symptoms, and consequently worsen renal function before beneficial effects are seen. Although carvedilol mainly undergoes hepatic metabolism, plasma levels will be elevated in patients with renal failure. For these reasons, it was deemed necessary to monitor renal parameters following the initiation and final titration of the agent.

One of the limitations of the present study is that all of the panellists were selected locally. This was done because the protocols were developed for use and local panellists would be most familiar with clinical practices and pharmacotherapies in Malaysia. Taking this into consideration, this 'limitation' may in fact be considered a strength of the present study (since the sampled panellists would best reflect the local management views and practices). Furthermore, as the titration protocols were developed based on international guidelines, it allowed for the exclusion of international panellists. In fact, based on the panellists' consensus, the final titration protocols were quite similar to those of various international guidelines. One other limitation was the absence of a face-to-face meeting among the panellists. A face-to-face meeting may have been beneficial for clarifying and working out any issues found within the titration protocols prior to their implementation. However, due to the panellists' hectic schedules and the study design, such a meeting was not possible.

In conclusion, the present Delphi study consulted a panel of experts, comprising cardiologists, physicians and pharmacists. The panel was able to achieve a consensus, which resulted in a set of validated drug titration protocols for the use of ACE inhibitors, ARBs, beta-blockers and spironolactone in patients with HF. These protocols can be used as a guide for pharmacists who need to initiate and titrate HF drug therapy in daily clinical practice. The recommendations of the pharmacists should be made in collaboration with the treating clinician.

\section{REFERENCES}

1. Kannel WB, Belanger AJ. Epidemiology of heart failure. Am Heart J 1991; 121:951-7.

2. Pitt B, Zannad F, Remme WJ, et al. The effect of spironolactone on morbidity and mortality in patients with severe heart failure. Randomized Aldactone Evaluation Study Investigators. N Engl J Med 1999; 341:709-17.

3. Packer M, Fowler MB, Roecker EB, et al. Effect of carvedilol on the morbidity of patients with severe chronic heart failure: results of the carvedilol prospective randomized cumulative survival (COPERNICUS) study. Circulation 2002; 106:2194-9.

4. Pfeffer MA, McMurray JJ, Velazquez EJ, et al. Valsartan, captopril, or both in myocardial infarction complicated by heart failure, left ventricular dysfunction, or both. N Engl J Med 2003; 349:1893-906.

5. McMurray J, Ostergren J, Pfeffer M, et al. Clinical features and contemporary management of patients with low and preserved ejection fraction heart failure: baseline characteristics of patients in the Candesartan in Heart failure-Assessment of Reduction in Mortality and morbidity (CHARM) programme. Eur J Heart Fail 2003; 5:261-70.

6. Cleland JG, Cohen-Solal A, Aquilar JC, et al. Management of heart failure in primary care (the IMPROVEMENT of Heart Failure Programme): an international survey. Lancet 2002; 360:1631-9.

7. Komajda M, Follath F, Swedberg K, et al. The EuroHeart Failure Survey programme--a survey on the quality of care among patients with heart failure in Europe. Part 2: treatment. Eur Heart J 2003; 24:464-74.

8. Luzier AB, Forrest A, Feuerstein SG, Schentaq JJ, Izzo JL Jr. Containment of heart failure hospitalizations and cost by angiotensin-converting enzyme inhibitor dosage optimization. Am J Cardiol 2000; 86:519-23.

9. Pearson GJ, Cooke C, Simmons WK, Sketris I. Evaluation of the use of evidence-based angiotensin-converting enzyme inhibitor criteria for the treatment of congestive heart failure: opportunities for pharmacists to improve patient outcomes. J Clin Pharm Ther 2001; 26:351-61.

10. Bucci C, Jackevicius C, McFarlane K, Liu P. Pharmacist's contribution in a heart function clinic: patient perception and medication appropriateness. Can J Cardiol 2003; 19:391-6.

11. Jain A, Mills P, Nunn LM, et al. Success of a multidisciplinary heart failure clinic for initiation and up-titration of key therapeutic agents. Eur J Heart Fail 2005; 7:405-10.

12. Hasson F, Keeney S, McKenna H. Research guidelines for the Delphi survey technique. J Adv Nurs 2000; 32:1008-15.

13. Slaughter A, Katz RV, Grasso JE. Professional attitudes toward denture adhesives: A Delphi technique survey of academic prosthodontists. J Prosthet Dent 1999; 82:80-9.

14. Hsu CC, Sandford BA. The Delphi Technique: Making Sense of Consensus. Pract Assess Res Eva [serial online] 2007; 12:1-8. Available at: http:// pareonline.net/getvn.asp? $v=12 \& n=10$. Accessed December 4, 2014.

15. Castagno D, Jhund PS, McMurray JJ, et al. Improved survival with bisoprolol in patients with heart failure and renal impairment: an analysis of the cardiac insufficiency bisoprolol study II (CIBIS-II) trial. Eur J Heart Fail $2010 ; 12: 607-16$.

16. Ghali JK, Wikstrand J, Van Veldhuisen DJ, et al. The influence of renal impairment on clinical outcome and response to beta-blockade in systolic heart failure: insights from Metoprolol CR/XL Randomized Intervention Trial in Chronic HF (MERIT-HF). J Card Fail 2009; 15:310-8.

17. Cice G, Ferrara L, D'Andrea A, et al. Carvedilol increases two-year survival in dialysis patients with dilated cardiomyopathy: a prospective, placebocontrolled trial. J Am Coll Cardiol 2003; 41:1438-44.

18. López-Sendón J, Swedberg K, McMurray J, et al. Expert consensus document on beta-adrenergic receptor blockers. Eur Heart J 2004; 25:1341-62.

19. Klotz U. Antiarrhythmics: elimination and dosage considerations in hepatic impairment. Clin Pharmacokinet 2007; 46:985-96.

20. Bakris GL, Weir MR. Angiotensin-converting enzyme inhibitor-associated elevations in serum creatinine: is this a cause for concern? Arch Intern Med 2000; 160:685-93.

21. Go AS, Rao RK, Dauterman KW, Massie BM. A systematic review of the effects of physician specialty on the treatment of coronary disease and heart failure in the United States. Am J Med 2000; 108:216-26.

22. Edep ME, Shah NB, Tateo IM, Massie BM. Differences between primary care physicians and cardiologists in management of congestive heart failure: relation to practice guidelines. J Am Coll Cardiol 1997; 30:518-26.

23. National Institute for Health and Clinical Excellence. Chronic Heart Failure: Management of Chronic Heart Failure in Adults in Primary and Secondary Care (NICE Clinical Guideline 108). London: National Institute for Health and Clinical Excellence, 2010. 